\title{
Erratum: Orthogonal micro-organization of orientation and spatial frequency in primate primary visual cortex
}

Ian Nauhaus, Kristina J Nielsen, Anita A Disney \& Edward M Callaway

Nat. Neurosci. 15, 1683-1690 (2012); published online 11 November 2012; corrected after print 11 January 2013

In the version of this article initially published, in the equation for $A_{\theta}$ on p. 5, the subscript to the variable $f$ was given as an $e$. The correct character is $\theta$. The error has been corrected in the HTML and PDF versions of the article.

\section{Erratum: Evolution of GluN2A/B cytoplasmic domains diversified vertebrate synaptic plasticity and behavior}

Tomás J Ryan, Maksym V Kopanitsa, Tim Indersmitten, Jess Nithianantharajah, Nurudeen O Afinowi, Charles Pettit, Lianne E Stanford, Rolf Sprengel, Lisa M Saksida, Timothy J Bussey, Thomas J O’Dell, Seth G N Grant \& Noboru H Komiyama

Nat. Neurosci. 16, 25-32 (2013); published online 2 December 2012; corrected online 10 December 2012 (details online); corrected after print 7 March 2013

In the version of this article initially published, the citations to Figures $3 a, 3 b$ and $3 c$ should have read $3 a-d, 3 e-h$ and $3 i-1$, respectively, and the citations to Figures $4 \mathrm{a}, 4 \mathrm{~b}$ and $4 \mathrm{c}$ should have read $4 \mathrm{a}-\mathrm{d}, 4 \mathrm{e}-\mathrm{h}$ and $4 \mathrm{i}-\mathrm{l}$, respectively. Also, under Gene Targeting in Online Methods, the incorporation of a GluN2A intronic fragment was omitted from the description of the GluN2B C-terminal exon construct. The sentence should read "The GluN2B C-terminal exon was amplified by PCR from 129/OlaHsd mouse genomic DNA (using primers 5'-GTATACACGGAGTAGCTATAGAGGAGCG-3' and 5'-GTTTAAACTCAGACATCAGACTCAATACTAGAAA-3') and attached to a small fragment of GluN2A intronic DNA (amplified using primers 5'-GGCGCGCCTAGGGCATCAATGACAGGG-3' and 5'- GTATACAACTGTAGATGCCCTGTGAGGG-3'), and the assembled product was inserted into AscI and PacI engineered to lie between the homology arms and the $3^{\prime}$ exon." The errors have been corrected in the PDF and HTML versions of this article.

\section{Corrigendum: The organization of two new cortical interneuronal circuits}

\author{
Xiaolong Jiang, Guangfu Wang, Alice J Lee, Ruth L Stornetta \& J Julius Zhu \\ Nat. Neurosci. 16, 210-218 (2013); published online 13 January 2013; corrected after print 3 March 2013
}

In the version of this article initially published, in Figure 8a under L1 Spontaneous, the last $400 \mathrm{~ms}$ of trace 4 was a duplicate of trace 3; for Figure 8c, the legend referred to a scale bar of $2 \mathrm{mV}$ instead of $4 \mathrm{mV}$; in Figure 8e, incidence on the $y$ axis was plotted in units of $0-0.8 \mathrm{~Hz}$ instead of $0-4 \%$; and in Figure $7 \mathrm{~b}$ the insets were not described. The insets show the sequences of soma/axon-dendrite-soma/axon-initiated events in the dendritic complex spikes at a timescale expanded by a factor of 2.5 , with arrows indicating the timing of initiation of the dendritic slow potentials and second somatic action potentials. The errors have been corrected in the HTML and PDF versions of the article.

\section{Corrigendum: Erbin interacts with TARP $\gamma$-2 for surface expression of AMPA receptors in cortical interneurons}

Yanmei Tao, Yong-Jun Chen, Chengyong Shen, Zhengyi Luo, C Ryan Bates, Daehoon Lee, Sylvie Marchetto, Tian-Ming Gao, Jean-Paul Borg, Wen-Cheng Xiong \& Lin Mei

Nat. Neurosci. 16, 290-299 (2013); published online 27 January 2013; corrected after print 30 July 2013

In the version of this article initially published, the email address for correspondence was given as lmei@grc.edu. The correct address is lmei@gru.edu. The error has been corrected in the HTML and PDF versions of the article.

\section{Erratum: Dopamine and the cost of aging}

Daphna Shohamy \& G Elliot Wimmer

Nat. Neurosci. 16, 519-521 (2013); published online 25 April 2013; corrected after print 13 May 2013

In the version of this article initially published, author G. Elliott Wimmer's name was misspelled. The error has been corrected in the HTML and PDF versions of the article. 